AperTO - Archivio Istituzionale Open Access dell'Università di Torino

\title{
Selective targeted delivery of TNFalpha to tumor blood vessels
}

\section{This is the author's manuscript}

Original Citation:

Availability:

This version is available http://hdl.handle.net/2318/1652551

since 2017-11-22T08:42:21Z

Published version:

DOI:10.1182/blood-2003-04-1039

Terms of use:

Open Access

Anyone can freely access the full text of works made available as "Open Access". Works made available under a Creative Commons license can be used according to the terms and conditions of said license. Use of all other works requires consent of the right holder (author or publisher) if not exempted from copyright protection by the applicable law. 
Leonardo Muratori, Anna La Salvia, Paola Sperone, Massimo Di Maio

Target therapies in recurrent or metastatic head and neck cancer: state of the art and novel perspectives. A systematic review.

\begin{abstract}
Recurrent or metastatic head and neck squamous-cell carcinomas (R/M HNSCC) are a group of cancers with a very poor prognosis. Many clinical trials testing novel target therapies in this setting are currently ongoing. We performed a systematic review focusing our attention on all clinical trials, ongoing or already published, concerning the use of novel drugs for treatment of R/M HNSCC. We found that the research of novel molecules effective in treatment of R/M HNSCC has been intense during last decade, and nowadays it is still very active. Unfortunately, the results in this setting have been, overall, disappointing: until now, only cetuximab and, recently, nivolumab and pembrolizumab received authorization for treatment of R/M HNSCC. Nevertheless, the promising results showed by some novel drugs may lead to continue the research in this field, with the aim of producing more evidence and finding new therapeutic indication.
\end{abstract}

Keywords: Head; Neck; Metastatic; Recurrent; Target; Immunotherapy.

\title{
1 - Introduction
}

Head and neck tumors are the fifth most common malignancies worldwide, and the sixth most common cause of cancer-related deaths [1]. More than 90\% of these tumors are squamous-cell carcinomas, and unfortunately most patients present with locally advanced disease, involving regional nodes or with distant metastases. Platinum-based chemotherapy still represents the standard first-line treatment for recurrent or metastatic head and neck squamous-cell carcinomas (R/M HNSCC) [2]. For subsequent lines, although several chemotherapeutical drugs have been tested during the last decades, the results have been globally disappointing, and overall survival for patients progressing after failure of platinum-based chemotherapy remains unacceptably poor. In the last few years, many novel targeted agents have been tested in many cancers, obtaining important results in several settings. Most of these molecules have been already tested in R/M HNSCC, and currently the number of clinical trials testing the activity of novel drugs in this setting is growing up. We concentrated the attention on those trials and we built a systematic review 
to describe the state of art of target therapy in this setting and of novel possible strategies for the future.

\section{2 - Materials and methods}

In September 2018, we conducted a systematic research with PubMed, using the following keywords: "metastatic", "recurrent", "head", "neck" and "cancer". We considered all the interventional studies concerned target therapy in R/M HNSCC published in English from January 2003 to July 2018, founding a total of 1806 publication from which we selected a final pool of 96 articles. Alongside, in September 2018, we searched in clinicaltrials.gov the following category: "Head and Neck Cancer", "Recurrent" and "Metastatic", founding 312 interventional studies with the same characteristics. Studies concerning target therapies in nasopharyngeal cancers and salivary gland cancers were also included but, based on the great differences in terms of natural history, prognosis and therapeutic option compared to HNSSC, they have been presented and discussed in a separated section.

\section{3 - EGFR inhibitors}

\section{1 - Cetuximab}

The drug most frequently tested in this setting is Cetuximab (Erbitux ${ }^{\text {TM }}$, Merck), a recombinant human-mouse chimeric IgG1 monoclonal antibody, that binds the epithelial growth factor receptor (EGFR) with high affinity, blocking an essential pathway for growth and survival of tumor cells. We found a total of 28 studies which tested cetuximab in R/M HNSCC. The drug was initially tested as monotherapy in a phase II single arm trial, in patients who had failed a platinum-based first line chemotherapy, showing an overall response rate (ORR) of $13 \%$ and a disease control rate (DCR) of $46 \%$, with a good toxicity profile [3]. The drug has been initially studied in combination with chemotherapy in phase II single arm studies [4-5], with encouraging results, but the first phase III trial, where the drug was tested versus placebo in platinum-pretreated R/M HNSCC, did not reach the statistical significance in terms of its primary end-point (median progression-free survival [PFS] was 4.2 months for cetuximab group versus 2.7 months for cisplatin-alone group; Hazard Ratio [HR] 0.80; 95\% C.I. 0.64 - 0.99; P=0.04) [6]. In 2008, the results of another phase III randomized study, the EXTREME trial, were published [7]. A total of 442 patients with previously untreated R/M HNSCC were randomized to receive either chemotherapy (5-FU + cisplatin / carboplatin) with cetuximab or the same chemotherapy without cetuximab. Adding cetuximab to platinum-based chemotherapy showed to significantly 
prolong overall survival (median OS was 10.1 months for cetuximab group versus 7.4 months for chemotherapy-alone group; HR 0.80; 95\% C.I. 0.64 - 0.99; P=0.04), with an acceptable tolerability and safety. Based on these results, the combination 5-FU + cisplatin/carboplatin + cetuximab became the standard for first-line treatment of R/M HNSCC. Furthermore, cetuximab was tested in combination with different chemotherapy drugs, but only in phase I and phase II, and not within randomized trials. As a matter of fact, no other phase III randomized trials have been published in last decade. Namely, the combinations of cetuximab with paclitaxel or docetaxel were both tested in phase II single arm trials, showing that the addition of the anti-EGFR antibody to a taxane is active and well tolerated (respectively, ORR 54\% and DCR $80 \%$ for the combination of paclitaxel + cetuximab in first line, and ORR 11\% and DCR 51\% for the combination of docetaxel + cetuximab in first and second lines) [8-9]. When cetuximab has been tested in combination with cisplatin and docetaxel, the combination produced an interesting ORR of 44\% [10]. The use of oxaliplatin instead of cisplatin in combination with 5-FU and cetuximab was studied in a phase I trial, showing acceptable kinetics profile and toxicity [11]. The most recent combination tested in a phase $\mathrm{II}$, single arm trial for previously untreated R/M HNSCC was cetuximab + cisplatin + pemetrexed, but it showed an unacceptable incidence of grade 3/4 toxicity and treatment-related deaths [12]. As of July 2018, 67 studies including cetuximab were ongoing. Since cetuximab is a standard of care in R/M HNSCC, in most cases it is used as comparator for phase II randomized trials which investigate novel drugs. Ten studies (mostly phase II) are investigating combinations of cetuximab and chemotherapy regimens (Table 1). A phase III trial is comparing first line single-agent methotrexate versus single-agent cetuximab in elderly patients (70 years and older) with R/M HNSCC (NCT01884623). The results of this trial could be important because, in the subgroup analysis of EXTREME trial, the addition of cetuximab to platinum-based chemotherapy did not demonstrate any survival benefit, compared with chemotherapy alone, in the subgroup of elderly patients, then nowadays it is difficult to identify a safe and effective standard for first line therapy in this setting of patients. Cetuximab was tested in R/M HNSCC even in combination with other target drugs, as will be discussed in the following sections (Table 2).

\section{2 - Panitumumab}

Panitumumab (Vectibix ${ }^{\mathrm{TM}}$, Amgen) is a humanized IgG2 monoclonal antibody, directed, as cetuximab, against EGFR. In particular, panitumumab is able to inhibit the 
autophosphorylation of receptor normally induced by its ligands, promoting the internalization of receptor and inhibiting the cellular growth. Even though this pathway is relevant in the biology of head and neck cancers, as demonstrated by the results obtained by cetuximab in recurrent or metastatic disease, the literature about this drug in this setting is lacking. Only one trial investigated the role of panitumumab with platinum-based chemotherapy as first line treatment in R/M HNSCC, the SPECTRUM trial [13]. This study randomized 657 patients to receive chemotherapy with cisplatin and 5-FU, alone or in combination with panitumumab. In the experimental group, patients could continue panitumumab in maintenance therapy. The study was negative, because the primary endpoint, OS, did not reach statistical significance (median OS was 11.1 months for experimental group versus 9.0 months for chemotherapy-alone group; HR 0,873; 95\% C.I. 0.729-1.046; $P=0.1403$ ), although the advantage in term of PFS was statistically significant (median PFS 5.8 months for panitumumab group versus 4.6 months for chemotherapyalone group; HR 0.780; 95\% C.I. 0.659-0.922; P=0.0036). Another first line randomized trial tested panitumumab in association with docetaxel and cisplatin, with similar results: statistically significant advantage in term of PFS (median 6.9 months for panitumumab group versus 5.5 months for chemotherapy-alone group; HR 0.629; 95\% C.I. 0.395-1.002; $\mathrm{P}=0.048$ ), but no effect in term of OS (median 12.9 months for panitumumab group versus 13.8 months for chemotherapy-alone group; HR 1.103; 95\% C.I. 0.709-1.717) [14]. The drug has been tested even in second line therapy after platinum progression, with disappointing results [15-16]. Considering these formally negative results, panitumumab has not been approved for the treatment of previously untreated R/M HNSCC. Despite the signals of activity, these negative results have discouraged further research and, as of July 2018, we found only four ongoing phase II studies.

\section{3 - Gefitinib}

Gefitinib (Iressa ${ }^{\mathrm{TM}}$, AstraZeneca) is the first small molecule that binds the tyrosine kinase domain of EGFR, and it is also the first biological drug tested in R/M HNSCC. In 2003, the results of a phase II single arm trial were published: 52 patients pretreated with no more than one line of chemotherapy were enrolled for treatment with gefitinib, and ORR and DCR were assessed, resulting respectively $10.6 \%$ and 53\% [17]. The drug was well tolerated and a statistically significative correlation between incidence of skin toxicity and response was found. In the wake of these results, several phase II clinical trials with gefitinib have been conducted in the last decade, but they resulted all negative [18-19-20- 
21]. Only two phase III trials were conducted. In the first one, gefitinib $250 \mathrm{mg} / \mathrm{day}$ and 500 $\mathrm{mg} / \mathrm{day}$, have been compared with methotrexate, without any OS benefit (median OS was, respectively, 5.6, 6.0, and 6.7 months; HR 1.22; 95\% C.I. 0.95-1.57; P=0.12; and HR 1.12; 95\% C.I. 0.87-1.43; $P=0.39$, respectively for 250 and $500 \mathrm{mg}$ vs methotrexate) [22]. The other study tested, in a double-blind assessment, the combination of docetaxel and gefitinib versus docetaxel and placebo, with similar results [23]. Considering all these negative results, gefitinib has not been approved for treatment of R/M HNSCC, and our research did not find any ongoing study with this drug in this setting.

\section{4 - Erlotinib}

Erlotinib (Tarceva ${ }^{\mathrm{TM}}$, Roche) is another anti-EGFR small molecule, very similar to Gefitinib in terms of structure, mechanism of action and kinetics. In phase I and II trials, it showed a modest activity in patients with R/M HNSCC, alone (ORR 4.3\%) [24], in combination with cisplatin (ORR 21\%) [25], or with bevacizumab (ORR 15\%) [26]. In addition, considering the affinity between EGFR an mTOR pathways, erlotinib has been studied in combination with mTOR inhibitors temsirolimus and everolimus, with disappointing results (that will be detailed in the section dedicated to mTOR inhibitors). No phase III trial concerning erlotinib in R/M HNSCC has been published, and only one can be found in the ClinicalTrials.gov: the study has the aim to investigate the effect of erlotinib in combination with paclitaxel and cisplatin or carboplatin as first line therapy for patients with R/M HNSCC, but it was early terminated because of low accrual (NCT00448240).

\section{5 - Lapatinib}

Lapatinib (Tykerb ${ }^{\mathrm{TM}}$, Novartis) is a tyrosine kinase inhibitor active on human epidermal growth factor receptor 2 (HER2) and EGFR. It was tested in a single-arm phase II trial, but although well tolerated, it appeared completely inactive in R/M HNSCC (ORR 0\%) [27].

\section{6 - Other monoclonal antibodies}

The family of EGFR inhibitors has recently been enriched by novel molecules, and some of them have been studied in R/M HNSCC. The only monoclonal antibody tested in this setting was zalutumumab, a fully human IgG1 directed against EGFR. It has been studied in second line after platinum progression, versus BSC, but did not increase OS (median 6.7 versus 5.2 months; HR 0.77, 95\% C.I. 0.57-1.05; $\mathrm{P}=0.0648$ ), even though it gave a limited, but statistically significative, benefit of PFS (median 9.9 versus 8.4 weeks; HR 
0.63, 95\% C.I. 0.47-0.84; $\mathrm{P}=0.0012$ ) [28]. An open-label, single-arm, phase II trial confirmed the limited activity of zalutumumab in this setting [29]. Duligotuzumab, a humanized IgG1 that inhibits selectively HER3, was assessed in a phase I trial in combination with chemotherapy, showing a good toxicity profile [30]. Tomuzotuximab, an optimized by fully glycosylation cetuximab, was under investigation in a randomized phase II trial, which compared the novel drug with cetuximab, both in combination with platinumbased chemotherapy, as first line treatment of R/M HNSCC (NCT02052960). At ASCO 2018 Congress a phase II randomized placebo-controlled study assessing the activity of patritumab, a fully human monoclonal antibody that blocks HER3, were presented (NCT02633800). The addition of patritumab to cisplatin/carboplatin and cetuximab produced an increase in toxicity, but the PFS of two arms was similar.

\section{7 - Other tyrosine-kinase inhibitors}

Among small molecules tyrosine-kinase inhibitors, both dacomitinib and afatinib were tested in R/M HNSCC. Dacomitinib has been studied within phase II trials in both first and second line, with encouraging results in terms of activity (ORR 12.7\% and 20.8\%, respectively) [31-32]. Afatinib showed antitumoral activity comparable with cetuximab in an exploratory phase II trial [33], and then it was tested in the LUX-Head \& Neck 1 trial: in this open label, randomized, phase III trial, 483 patients with R/M HNSCC experiencing disease progression after platinum-based chemotherapy were randomly assigned to receive either afatinib or methotrexate, and PFS was assessed as primary endpoint [34]. Afatinib resulted superior to chemotherapy (median PFS was 2.6 months for the afatinib group versus 1.7 months for the methotrexate group; HR 0.80; 95\% C.I. 0.65-0.98; $\mathrm{P}=0.030$ ), and well tolerated. Furthermore, in a post-hoc subgroup analysis of this study, it was showed that the cluster of elderly patients ( $\geq 65$ years) seem to obtain a similar benefit compared to their younger counterpart, without a relevant increase in toxicity [35]. However, although these interesting features and even though the study formally reached his primary end-point, the limited clinical magnitude of the result did not lead to the regulatory approval of the drug in this setting. As of July 2018, a phase II study testing the double EGFR inhibition with the combination of cetuximab and afatinib was ongoing (NCT02979977).

\section{4 - Angiogenesis inhibitors}

4.1 - Sorafenib 
Sorafenib (Nexavar ${ }^{\mathrm{TM}}$, Bayer) is a small molecule tyrosine-kinase inhibitor that blocks the tumor growth through the inhibition of multiple targets. In detail, this drug targets the angiogenesis, an essential process for the development of tumor microenvironment, due to the inhibition of vascular endothelial growth factor receptors 1, 2 and 3 (VEGFR1, VEGFR2 and VEGFR3). Other targets include platelet-derived growth factor receptor (PDGFR), rapidly accelerated fibrosarcoma kinases $B$ and $C$ (B-RAF and C-RAF) and hepatocyte growth factor receptor (HGFR, also called c-MET). In R/M HNSCC, the drug was studied only in phase II trials, as single agent [36-37-38-39], or in combination with cetuximab [40], showing a limited activity, regardless of the number of prior therapies.

\section{2 - Bevacizumab}

Bevacizumab (Avastin ${ }^{\mathrm{TM}}$, Roche) is a humanized monoclonal antibody, that blocks the angiogenesis from the extracellular side, binding directly the VEGF-A molecule and preventing its interaction with VEGFR 2. The drug was initially tested in R/M HNSCC in combination with other drugs, but only in single-arm, non-randomized phase I and II trials. In combination with erlotinib, bevacizumab resulted well tolerated, but the efficacy in term of ORR (15\%) was limited, as discussed above. The combination of pemetrexed and bevacizumab was assessed as first line therapy in patients with R/M HNSCC, showing an interesting TTP of 5 months and a DCR of $86 \%$, but even resulting poorly tolerated, in particular in term of bleeding events (6 of 40 patients developed a grade 3 or worse bleeding, and two of these were fatal) [41]. At 2017 ASCO meeting, the results of a randomized phase III trial were presented: 403 patients with previously untreated R/M HNSCC were randomized to receive either chemotherapy of investigator's choice plus bevacizumab or chemotherapy alone. With a median follow-up of 23.1 months, the addition of bevacizumab did not give a statistically significative advantage of OS, that was the primary endpoint of the study: median OS was 12.6 in bevacizumab arm and 11.0 months in control arm (95\% Cl 0.67-1.05; $\mathrm{P}=0.13)$.

Bevacizumab was studied even in combination with cetuximab, with encouraging results in term of response and preliminary outcome parameters in previously treated patients [42].

\section{3 - Sunitinib}

Sunitinib (Sutent ${ }^{\mathrm{TM}}$, Pfizer) is a multitargeted kinase inhibitor that inhibits several receptor tyrosine kinases, in particular VEGFR 1, VEGFR 2, PDGFR-alpha, PDGFR-beta, KIT and FLT3. This activity results in a block of angiogenesis, followed by an inhibition of tumor 
growth. Only three phase II trials were conducted in R/M HNSCC, and unfortunately the results in term of response and safety were very disappointing [43-44-45]. These negative results have discouraged further investigation.

\section{4 - Other drugs}

Semaxanib was initially studied in a phase I trial in combination with paclitaxel, showing a negative safety profile [46], and thereafter it was tested as single-agent in a phase II single arm trial, resulting in a ORR of $6 \%$ (the unfavorable safety toxicity profile was confirmed even as single-agent) [47]. Axitinib was tested in a single arm phase II study, showing an interesting efficacy and a good safety profile as first line treatment (DCR 76\%) [48]. Based on these preliminary encouraging results, the same teamwork launched an expansion trial (NCT02762513), that is ongoing as of July 2018. Finally, vandetanib was tested in a randomized phase II trial, in combination with docetaxel, versus docetaxel alone [49]. Although the addition of target therapy was associated with a numerical improvement in terms of response and PFS, the difference between arms was not statistically significant, and the study was formally negative (ORR: 13\% for the combination versus $7 \%$ for chemotherapy alone; PFS 9.0 weeks for the combination versus 3.2 weeks for chemotherapy alone). Concerning apatinib, no trial has been published, but a trial which investigated the combination apatinib/tegafur in patients with R/M HNSCC was ongoing (NCT03096184). At 2018 ASCO meeting, results of a pilot trial which assessed the efficacy of apatinib in pretreated R/M HNSCC were presented (NCT03424291). 16 patients were enrolled and palliative radiotherapy $(R T)$ was given to patients who could tolerate the combination (8 patients). ORR was 25\% for apatinib alone and 87,5\% for apatinib and RT; DCR was 50\% for apatinib alone and 100\% for apatinib and RT.

\section{5 - mTOR inhibitors}

\section{1 - Everolimus}

Everolimus (Afinitor ${ }^{\mathrm{TM}}$, Novartis) is a derived of rapamycin that inhibits MTOR, a regulation protein frequently overexpressed in human cancers. The activity of this drug in R/M HNSSC was studied within two phase I trials, which showed good kinetics profile and safety [50-51]. Then, a phase II trial was conducted in 2015, and everolimus was tested, in combination with erlotinib, in platinum-pretreated patients [52]. The results were disappointing (ORR 2.8\%; DCR 28.8\%). Some other phase II trials with everolimus have 
been subsequently started, but most of those trials were terminated prematurely, because of low accrual.

\section{2 - Temsirolimus}

Temsirolimus (Torisel ${ }^{\mathrm{TM}}$, Pfizer) is another rapamycin-derived with a mechanism of action and a kinetics very similar to everolimus. It was tested in R/M HNSCC in a phase I trial, in combination with paclitaxel and carboplatin, showing a good tolerability profile [53], and in combination with erlotinib in a phase II trial, with negative results [54]. In contrast to everolimus, another phase II trial with temsirolimus registered some interesting results: namely, 40 patients with platinum-pretreated R/M HNSCC were treated with temsirolimus, and an ORR of $39.4 \%$ and a DCR of $97 \%$ were measured, even though the PFS was only 2 months [55]. The phase II trial with temsirolimus in combination with paclitaxel and carboplatin (NCT01016769) was completed but still not published as of July 2018.

\section{6 - PI3K inhibitors}

\section{$6.1-\mathrm{PX}-866$}

PX-866 (Cascadian Therapeutics) in an oral irreversible inhibitor of PI3K, a signaling pathway strictly related with mTOR pathway and frequently altered in some human cancer. The drug was tested in a phase I trial in combination with cetuximab, showing a good kinetics profile and with promising results in term of response (ORR 44\%, DCR 88\%) in patients with R/M HNSCC [56]. Unfortunately, the subsequent randomized phase II trial did not confirm the hypothesis: in combination with cetuximab [57], as well as in combination with docetaxel [58], PX-866 did not produce any evidence of benefit in term of PFS (median was 80 days for the combination versus 80 days for cetuximab alone in the first trial; 92 days for the combination versus 82 days for docetaxel alone in the second trial).

\section{2 - Buparlisib}

Buparlisib (Novartis) is another new molecule that binds PI3K inhibiting tumor cell growth and survival. The preclinical features of the molecule were studied in a phase I trial conducted in a group of patients with different kinds of metastatic cancers, including HNSCC, and it showed a manageable safety profile [59]. In 2017, the results of a randomized phase II trial were published [60]. 158 patients with platinum-pretreated R/M HNSCC were randomly assigned to receive either buparlisib and paclitaxel or placebo and 
paclitaxel, and PFS and OS were assessed as primary and secondary endpoint, respectively. The addition of buparlisib gave a statistically significant (although clinically modest) benefit, with a median PFS of 4.6 months, while the median PFS of chemotherapy alone was 3.5 months (HR 0.65; 95\% C.I. 0.45-0.95; P=0.011). The median OS was 10,4 months for buparlisib group and 6.5 months for placebo group (HR 0.72; 95\% C.I. 0.49-1.04; $\mathrm{P}=0.041$ ). Despite the 35\% reduction in the risk of disease progression, the advantage associated with the addition of buparlisib was quite modest in absolute terms (slightly more than 1 month difference in median PFS), then a phase III trial to evaluate this combination has still not been conducted.

\section{3 - Other drugs}

SF1126 (SignalRx Pharmaceuticals) is a PI3K inhibitor, which was tested in a phase II study with the aim to assess its activity in R/M HNSCC. Unfortunately, the study was terminated early because of too slow enrollment (NCT02644122). Instead, two phase II trials which investigated the PI3K inhibitors alpelisib and copanlisib, both in patients with platinum-pretreated R/M HNSCC, were still ongoing (NCT02145312, NCT02822482) as of July 2018.

\section{7 - CDK inhibitors}

\section{1 - Palbociclib}

Palbociclib (Ibrance ${ }^{\mathrm{TM}}$, Pfizer) is a novel inhibitor of cyclin-dependent kinases (CDK) 4 and 6. In the setting of R/M HNSCC, only a phase I trial which investigated the combination palbociclib/cetuximab has been published [61]. The trial, designed to assess the kinetics of this combination, included 9 patients, one of them registered a partial response and three of them a disease stabilization. At 2018 ASCO meeting, an abstract selected for oral presentation presented the results of an ongoing phase I/II trial that investigated the same combination, palbociclib/cetuximab, in patients with platinum-resistant and cetuximabnaive R/M HNSCC. With 30 patients enrolled, ORR was 35\%, median PFS was 6.4 months and median OS was 12.1 months (NCT02101034). Other two studies were ongoing as of July 2018: another phase II trial investigating the combination palbociclib/cetuximab in R/M HNSCC (NCT02499120), and a phase II that investigated the combination palbociclib/carboplatin (NCT03194373). 


\section{2 - Other drugs}

Ribociclib (Kisqali ${ }^{\mathrm{TM}}$, Novartis) and abemaciclib (Verzenio ${ }^{\mathrm{TM}}$, Eli Lilly) are other checkpoint inhibitors, very similar to palbociclib in terms of mechanism of action. Both drugs were under evaluation for the treatment of R/M HNSCC in phase II trials: ribociclib in combination with cetuximab for patients with R/M HNSCC already treated with any number of therapy lines (NCT02429089), abemaciclib as single-agent for patients with platinumpretreated disease (NCT03356587). Even a last CDK inhibitor, P276-00, was under evaluation in a phase II trial (NCT00824343), completed but not published as of July 2018.

\section{8 - Other targeted therapies}

We found 17 target drugs not included in the categories exposed above, divided in 20 published studies: 4 phase I trials and 16 phase II trials.

\section{1 - Proteasome inhibitors}

The inhibition of proteasome determinates a block cellular breakdown of proteins, producing an accumulation of toxic substances in the cytoplasm and the activation of apoptosis. Bortezomib (Velcade ${ }^{\mathrm{TM}}$, Janssen) is a proteasome inhibitor, already used in clinical practice for treatment of multiple myeloma. It was tested in combination with docetaxel in patients with R/M HNSCC who had received no more than one prior line of therapy, showing a high response rate (ORR 52\%) [62], but the subsequent randomized phase II trial, where bortezomib was tested in combination with irinotecan, did not confirm the positive results (ORR for the combination 13\% versus 3\% for bortezomib alone) [63]. Another proteasome inhibitor, ixazomib, was tested in a phase I trial, showing a good safety profile but a total lack of activity [64].

\section{2 - Multitarget inhibitors}

Dasatinib (Sprycel ${ }^{\mathrm{TM}}$, Bristol) is multitarget inhibitor that blocks many kinases including BCR-ABL, some kinases of SRC family, C-KIT, EPHR and PDGFR. It is currently approved for treatment of chronic myelogenous leukemia and acute lymphoblastic leukemia. The drug was tested in R/M HNSCC, but the results were disappointing [65]. On the contrary, foretinib, an inhibitor of VEGFR2, hepatocyte growth factor (HGF) and c-Met, was tested in a phase II trial giving promising results (ORR 42\%, DCR 50\%) [66].

\section{3 - Histone deacetylase inhibitors}


Romidepsin (Zolinza ${ }^{\mathrm{TM}}$, Merck) and vorinostat (Istodax ${ }^{\mathrm{TM}}$, Celgene) are two molecules that inhibit the histone deacetylase (HDAC), an enzyme that normally blocks the interaction between DNA and transcription factors, preventing gene expression. An altered activity of HDAC, which determinate the expression of wrong genes or the absence of expression of other essential genes, was detected in some human cancers. These drugs, already approved for the treatment of cutaneous T cell lymphoma, were tested in R/M HNSCC, but with negative results [67-68].

\section{4 - Toll-like receptors agonists}

Toll-like receptor family includes 13 receptors, numbered from TLR1 to TLR13. They are expressed on the membrane of macrophages and dendritic cells and have the task to recognize pathological epitopes form bacteria and other microbes that overtake the anatomic barriers. When stimulated, the subtype TLR9 seems to be implied with the production of cytokines with antiproliferative and antitumor activity. EMD1201081 is a TLR9 agonist that was tested in combination with cetuximab versus cetuximab alone as second line therapy for patients with platinum-pretreated R/M HNSCC, not showing any survival benefit [69]. Another TLR9 agonist, IMO-2055, was studied in combination with platinum-based chemotherapy in a phase I trial, showing a bad safety profile [70]. A TLR8 agonist, VTX-2337, was under investigation in a phase II trial, in combination with cetuximab (NCT01334177), completed but not published as of July 2018.

\section{5 - Other mechanisms}

Other eight drugs were evaluated in R/M HNSCC, but no one showed a level of activity that could justify further investigations. The CD44 inhibitor bivatuzumab and the PKC-beta inhibitor enzastaurin were evaluated only in phase I studies designed to assess the kinetics features of the drugs [71-72]. Ispinesib (KSP inhibitor), lonafarnib (farnesyltransferase inhibitor), sacaratinib (SRC inhibitor), figitumumab (IGF-1 inhibitor), dovitinib (FLT-3 inhibitor) and dalantercept (ALK-1 inhibitor) were tested in single-arm phase II trials, but no one of them showed any acceptable level of antitumoral activity in R/M HNSCC [73-74-75-76-77-78].

\section{9 - Immunotherapy}

Over the last years, immunotherapy has been intensively studied as a potential treatment for cancer [79]. Cancer immunotherapy comprehends a great variety of treatment 
approaches, including active immunotherapeutic strategies, such as cancer vaccines, and passive immunotherapies, such as monoclonal antibodies or adoptive transfer of tumorspecific $T$ cells. Tumor cells often present multiple resistance mechanisms that allow their escape from the host-tumor immune system [80]. Programmed cell death-1 (PD-1) is an inhibitory receptor expressed on activated $\mathrm{T}$ and $\mathrm{B}$ cells, which normally function to dampen the immune response [81]. PD-1 is engaged by ligands PD-L1 and PD-L2, which are expressed by tumor cells and infiltrating immune cells. Inhibition of the interaction between PD-1 and PD-L1 can enhance anti-tumor responses, delay tumor growth, and facilitate tumor rejection. Therefore, tumor cell destruction facilitated by immune checkpoint blockade is a strategy for cancer immunotherapy [82].

\section{1 - Nivolumab}

Nivolumab (Opdivo ${ }^{\mathrm{TM}}$, Bristol) is human IgG4 monoclonal antibody, which binds PD-1 protein. A multinational, multicenter, open-label, randomized phase III study, the Checkmate-141 trial, was conducted testing nivolumab as second line treatment in R/M HNSCC [83]. 361 patients who developed a progressive disease during or within 6 months of platinum-based chemotherapy were randomized (in a 2:1 ratio) to receive either nivolumab monotherapy $3 \mathrm{mg} / \mathrm{kg}$ or a single-agent therapy at investigator's choice (cetuximab, methotrexate or docetaxel). The primary endpoint was OS; secondary endpoints were PFS and ORR. Nivolumab resulted superior to standard therapy in terms of OS (median 7.5 months for nivolumab group versus 5.1 months for standard group; HR 0.70; 97.73\% C.I. 0.51- 0.96; $\mathrm{P}=0.01$ ), but not in terms of PFS (median 2.0 months for nivolumab group versus 2.3 months for standard group; HR 0.89; 95\% C.I., 0.70- 1.13; $\mathrm{P}=0.32$ ). Based on these results, Checkmate-141 was stopped early because the study met its primary endpoint of OS and nivolumab was approved by European Medicines Agency in 2017 for second line treatment of R/M HNSCC, progressed after platinumbased therapy. Other studies were ongoing as of September 2018, and some of them were investigating the combination of nivolumab with other drugs. In particular, two randomized studies, a phase II and a phase III, were evaluating nivolumab in combination with ipilimumab (NCT02823574; NCT02741570). The first, CheckMate-741 trial, is a phase II study comparing nivolumab monotherapy with nivolumab plus ipilimumab. The second one, CheckMate-651, is a phase III trial comparing nivolumab and ipilimumab combination with standard chemotherapy (cetuximab plus cisplatin versus carboplatin plus 5fluorouracil) as first-line treatment in R/M HNSCC. Other two phase III have been designed 
to test the combination of nivolumab with the novel drugs BMS-986205 (NCT03386838) and epacadostat (NCT03342352), respectively, but both trials terminated early because of low accrual. Furthermore, a phase I/II trial has been designed to determine if the combination of cetuximab and nivolumab could be active in patients with R/M HNSCC (NCT03370276). Two phase I studies evaluated the association between nivolumab and new small molecules. The first one combined the anti PD-1 agent with the oncolytic virus enadenotucirev (NCT02636036). The second one enrolled patients with advanced cancers including R/M HNSCC to test the association of nivolumab and TAK-659, a selective, reversible, potent SYK/FLT-3 inhibitor (NCT02834247).

\section{2 - Pembrolizumab}

Pembrolizumab (Keytruda ${ }^{\mathrm{TM}}$, Merck) is another novel humanized monoclonal antibody, with a mechanism of action very similar to nivolumab, and it represents the first immunotherapy ever tested in HNSCC. In the first phase I trial, designed to assess the safety of the drug in patients with R/M HNSCC, pembrolizumab showed an ORR of $18 \%$ (including $4 \mathrm{CR}$ and $20 \mathrm{PR}$ ), with a median OS of 8 months and a 6-month PFS of 23\% [84]. These data led to the accelerated approval of pembrolizumab by the U.S. Food and Drug Administration (FDA). In 2017, the results of a single arm phase II trial were published: 171 patients with platinum pretreated R/M HNSCC were treated with pembrolizumab, and the response rate previously obtained in the phase I trial was substantially confirmed (ORR 16\%) [85]._Outcomes from the confirmatory KEYNOTE-040 phase III trial comparing pembrolizumab with standard therapies in patients with platinumresistant R/M HNSCC were presented at 2017 ESMO meeting [86], although the final published manuscript is still awaited. In this trial, 495 patients were enrolled, 247 were assigned to pembrolizumab $200 \mathrm{mg}$ every three weeks, 248 to standard therapies (65 methotrexate, 110 docetaxel, 73 cetuximab). As a result, ORR was higher with pembrolizumab (14.6\% versus $10.1 \%$ ), whereas there was no difference in PFS. Median OS was 8.4 months with pembrolizumab compared to 7.1 months in the control arm (HR 0.81; 95\% C.I. 0.66 - 0.99) but did not reach statistical significance $(P=0.020)$. However, based on these promising results, many ongoing studies are testing pembrolizumab. At 2018 ASCO Annual Meeting the results of the phase III, randomized, open-label KEYNOTE-048 (NCT02358031) have been presented. This 3-arm trial was designed to investigate pembrolizumab in the first-line setting (both as monotherapy and in combination with a platinum-based (cisplatin or carboplatin) chemotherapy plus 5- 
fluorouracil), compared to standard treatment with cetuximab plus platinum-based chemotherapy plus 5-fluorouracil in R/M HNSCC. The primary endpoints were OS and PFS, and the secondary endpoints of the study were PFS at 6 months and 12 months and ORR. Based on an interim analysis, the treatment with pembrolizumab monotherapy resulted in significantly longer OS compared to the control arm, that is the current standard of care for R/M HNSCC in the first-line treatment setting. At the time of the interim analysis, the dual-primary endpoint PFS for patients whose tumors expressed PD-L1 (CPS $\geq 20$ ) had not been reached. Another update of the study was presented at 2018 EMSO meeting. Pembrolizumab alone resulted superior to standard treatment in terms of OS in CPS $\geq 20$ (median 14.9 vs 10.7 months; HR 0.61; 95\% Cl 0.45-0.83; P=0.0007) and CPS $\geq 1$ (median 12.3 vs 10.3 months; HR 0.78; 95\% Cl 0.64-0.96; $\mathrm{P}=0.0086$ ) and non-inferior to in the total population. The combination of pembrolizumab and chemotherapy resulted superior to standard treatment in the total population (median 13.0 vs 10.7 months; HR $0.77 ; 95 \% \mathrm{Cl} 0.63-0.93 ; \mathrm{P}=0.0034)$. Although the study will continue to the final OS analysis, the authors suggest that the combination of pembrolizumab and chemotherapy could be taken into account as a new standard of care for first line treatment in R/M HNSCC.

A phase II study was assessing single-agent pembrolizumab, still in first line, but specifically in a population of frail, elderly and platinum-ineligible patients, versus methotrexate (NCT03193931). Another study was assessing the activity of pembrolizumab plus docetaxel (NCT02718820). Other studies were testing pembrolizumab in combination with new target agents. Two studies, a phase II (NCT03238638) and a randomized phase III (NCT03358472), were evaluating pembrolizumab in combination with epacadostat. Other ongoing trials were combining pembrolizumab with cetuximab (NCT03082534), cabozantinib (NCT03468218), vorinostat (NCT02538510), enoblituzumab (NCT02475213) and acalabrutinib (NCT02454179), respectively. Furthermore, a phase II study enrolling R/M HNSCC and locally advanced or metastatic non-small cell lung cancer (NCT03049618), explores the combination between pembrolizumab and a recombinant fusion protein, EphB4-HAS, composed of the full-length extracellular domain of human receptor tyrosine kinase ephrin type-B receptor 4 (sEphB4) and fused, at its C-terminus, to full-length human serum albumin (HSA), with potential antineoplastic and anti-angiogenic activities. Finally, a single arm phase II study was evaluating the efficacy of pembrolizumab alone, in the setting of unresectable, locally recurrent patients without distant metastases (NCT02769520). 


\section{3 - Others}

The recent approval of nivolumab (EMA and FDA) and pembrolizumab (by FDA) has provided significant clinical improvements in the treatment of HNSCC, offering new encouraging options in R/M HNSCC. However, alternative targets for immune checkpoint inhibition exist, in both PD-L1 and CTLA-4 axes. For the first category, durvalumab (Imfinzi ${ }^{\mathrm{TM}}$; AstraZeneca), an IgG1 antibody against PD-L1, has demonstrated to be active in different types of tumors, including HNSCC. 62 R/M HNSCC patients received durvalumab every 2 weeks intravenously at the dosage of $10 \mathrm{mg} / \mathrm{kg}$ for 12 months within an open-label phase I/II trial including multiple advanced solid malignancies (NCT01693562). Preliminary results of this study were presented at the 2016 ESMO meeting [87]. 6-month and 12-month OS were 62\% and 42\%, respectively, and responses were durable (>12 months in 6 out of 7 responders). Additionally, durvalumab is being evaluated as monotherapy in platinum-refractory, PD-L1-positive R/M HNSCC in a phase II trial (HAWK; NCT02207530). Preliminary results for 111 evaluated patients were recently presented at the 2017 ESMO meeting [88]. ORR was 16.2\%, median PFS was 2.1 months, median OS was 7.1 months. Finally, other trials testing the PD-L1 inhibitors atezolizumab and avelumab in HNSCC were ongoing, but no one specifically concerned the R/M setting.

As regards the second axis (CTLA-4), to date no results have been reported for the monoclonal antibody against CTLA-4 ipilimumab, in HSNCC. As already cited above, two ongoing studies are evaluating the combination of ipilimumab and nivolumab, that has been tested in many solid tumors and has already produced relevant results in several settings, including melanoma, renal cell cancer and non-small cell lung cancer. Moreover, another selective human IgG2 monoclonal antibody anti-CTLA-4 tremelimumab, was under study in combination with the antti-PD-L1 durvalumab. Namely, we found six trials testing this combination in R/M HNSCC: three were single-arm phase I/II trials (NCT03381183, NCT03019003, NCT02499328) which tested tremelimumab and durvalumab in combination with other novel drugs; the others were randomized trials, a phase II (NCT02319044) and two phase III (NCT02369874, NCT02551159), which compared the combination tremelimumab/durvalumab with the standard therapy.

\section{0 - Novel perspectives}


We found some ongoing studies which were investigating the efficacy of targeted drugs with novel mechanism of action in R/M HNSCC patients, and we selected those more relevant. Monalizumab is a humanized IgG4 that inhibits NKG2A, a receptor expressed on tumor infiltrating NK cells and CD8+ T cells that, binding HLA-E, blocks the activity of these cells against cancer. Preventing this interaction, the drug empowers immune response against tumor cells. A single arm phase II study, which tested the activity of combination monalizumab/cetuximab in patients with platinum-pretreated R/M HNSCC, was ongoing as of July 2018 (NCT02643550). Ficlatuzumab is novel antibody that binds the hepatocyte growth factor (HGF inhibitor), preventing the activation of c-Met pathway, strictly linked with tumor growth, angiogenesis and development of metastasis. As well as monalizumab, the drug was under investigation in a single arm phase II study, combined with cetuximab, in patients with platinum-pretreated R/M HNSCC (NCT02277197). A drug strictly related with ficlatuzumab in terms of molecular target is tivantinib, a small molecule that inhibits directly c-Met receptor, preventing the activation of this pathway. A randomized phase II trial was ongoing, evaluating the combination of tivantinib plus cetuximab versus cetuximab alone (NCT01696955). AZD9150 is a novel molecule that inhibits STAT3, a transcription factor belonging to STAT family, that can be constitutively active in some human cancers, promoting the tumor growth. AZD5069 is an inhibitor of CXCR2, a membrane receptor activated by CXC chemokine, also implicated in processes that stimulate tumor development. These two drugs were under investigation in a sevenarm randomized phase II trial in various combinations with durvalumab, for the treatment of R/M HNSCC (NCT02499328, already cited in immunotherapy section).

\section{1 - Nasopharyngeal and salivary gland cancers}

\section{1 - Nasopharyngeal cancers}

Nasopharyngeal cancers (NPC) are a subgroup of HNSCC arising from the mucosal of the nasopharynx. The incidence in developed countries is very low. Although usually the combination of chemotherapy and radiotherapy allows to achieve the complete disease eradication, the R/M disease is an less common but clinically challenging situation [89]. For these reasons, the studies which investigated novel target therapies in this setting are very few, and the results have been generally disappointed. Cetuximab was tested in combination with carboplatin in 60 patients with NPC who progressed on or within 12 months after termination of platinum-based chemotherapy, showing a modest activity (ORR 11.7\%, DCR 60.0\%). Moreover, an ongoing phase III trial was investigating the 
combination of cisplatin + docetaxel + cetuximab compared to docetaxel alone, in patients with metastatic NPC (NCT02633176), based on the results of the phase II study previously mentioned, which had showed the efficacy of this combination of chemotherapy and cetuximab. Finally, gefitinib was investigated in patients with NPC pretreated to at least two lines of chemotherapy, but no partial or complete response were observed [91].

\section{2 - Salivary gland cancers}

Salivary gland cancers of head and neck (SGCHN) are another uncommon type of cancer, originating from major and minor exocrine glands that product saliva [92]. A lot of different histologic subtypes have been described, but in clinical practice they are usually divided in adenoid cystic cancers (ACC) and non-adenoid cystic cancers (non-ACC). Although these cancers are uncommon, some histologies have a high risk of disease relapse after radical treatment (mucoepidermoid carcinoma, squamous cell carcinoma, anaplastic carcinoma). Although R/M disease is often considered chemosensitive, it remains an incurable condition with a poor prognosis then, with the limitations set by the rarity of these tumors, some clinical trials investigating novel target drugs have been carried out in this setting.

Cetuximab was tested in a group of 30 patients with R/M SGCHN (23 ACC and 7 nonACC), who had received no more than one prior chemotherapy [93]. The primary endpoint was clinical benefit rate (CBR), defined as the occurrence of objective response or stable disease for at least 6 months: stable disease was obtained in $50 \%$ of patients, but no objective response was observed. Nowadays cetuximab is not approved in this setting. Between EGFR-inhibitors, a phase II trial investigated gefitinib in R/M SGCHN, but the results were disappointing [94].

In 2016, the results of a phase II trial which tested sorafenib in R/M SGCHN were published [95]. 37 patients (19 ACC and 18 non-ACC) were treated with sorafenib 400 $\mathrm{mg} / \mathrm{die}$, showing an ORR of $50 \%$ and a DCR of $76 \%$, according to Choi criteria. Based on these results, R/M SGCHN represent the only setting of head and neck cancers group where sorafenib could deserve further investigation, but no trial is ongoing as of October 2018. The results of a single arm phase II trial which tested pazopanib in R/M SGCHN was presented at the 2017 ASCO meeting: 72 patients (49 ACC and 20 non-ACC) were treated with daily pazopanib $800 \mathrm{mg} / \mathrm{die}$, showing a stable disease rate of $76 \%$ and $72 \%$, respectively, although only two partial responses were observed. Median OS was not reached when these results have been presented (NCT02393820). At 2018 ASCO meeting, two phase II studies that assessed the efficacy of another angiogenesis-inhibitor, 
lenvatinib (Lenvima ${ }^{\mathrm{TM}}$, Eisai), were presented (NCT02780310, NCT02860936). In both studies, lenvatinib showed an interesting anticancer activity in R/M ACC (tumor size reduction $64 \%$ in the first one and $27 \%$ in the second). Globally, to date, sorafenib, pazopanib and lenvatinib are the only angiogenesis-inhibitors who showed promising results in this setting.

Other molecules, belonging to already mentioned categories, were tested specifically in R/M SGCHN, but the results have been globally disappointing. Nintedanib was studied for treatment of R/M SGCHN, but it did not result active at all (ORR 0\%) [96]. Bortezomib was assessed in a single arm phase II trial, but it resulted associated with no demonstration of activity (ORR 0\%) [97]. Dasatinib was tested in R/M SGCHN, but the results were not impressive (ORR 2.5\% in ACC patients and 0\% in non-ACC patients) [98]. Finally, imatinib, an old inhibitor of BCR-ABL and some other kinases, was tested in patients with R/M salivary gland cancers, showing a modest activity [99].

A novel category of target drugs that recently showed promising results is TRK-inhibitors. Tropomyosin receptor kinases $A, B$ and $C$ (TRKA, TRKB and TRKC) are proteins that regulate some aspects of pain transmission in central nervous system. The chromosomes that carry their genes often suffer fusion events, which hesitate in chimeric proteins, some of them with oncogene potential. Larotrectinib is an inhibitor of all three TRK fusion proteins, which recently was tested in a phase I-II trial [100]. The study was designed as a "basket trial", including 55 patients with different kinds of neoplasm, all carrying TRK fusion. A total of 12 patients had R/M SGCHN, and this was the most common histology included in the study. The objective response rate, primary endpoint of the study, in the

whole study population was $75 \%$. Based on these promising results, other studies with larotrectinib were ongoing (NCT02576431, NCT02122913), but the rarity of molecular target forces the researchers to choose the basket trial design, making hard to imagine a single-histotype study.

Concerning immunotherapy in R/M SGCHN, no clinical trials were published as of October 2018. Only a French study was recruiting patients to evaluate the efficacy of nivolumab in patients with R/M SGCHN (NCT03132038).

\section{2 - Discussion}

Head and neck tumors represent a disease with considerable clinical and therapeutic complexity, whose incidence is slowly increasing. The recurrent/metastatic disease represents a setting with a still poor prognosis, given that a few therapies have 
demonstrated efficacy in the first line setting, and even poorer is the therapeutic scenario for the second line. In recent years, the development of new molecular targeted therapies has undergone considerable acceleration, and therefore R/M HNSCC has become object of many studies, with the aim of evaluate the effectiveness of various molecules.

Without doubt, the great majority of the studies have not reached the expected results, in particular small molecules EGFR-inhibitors, angiogenesis-inhibitors and mTOR inhibitors, that nowadays are largely used in several types of cancers. In any case, this intensive activity of research resulted in the approval of three target drugs in this setting, cetuximab, nivolumab and pembrolizumab. However, most questions remain open in the treatment of R/M HNSCC.

An important problem is represented by the optimal treatment of elderly patients. As previously mentioned, in the subgroup analysis of the EXTREME study, which resulted in the approval of the cisplatin/5-FU/cetuximab scheme in first line, the cluster of patients over 70 years seemed not to obtain the same survival benefit of younger patients. Therefore, nowadays, this therapy is considered the standard for all age clusters, but without a real evidence of its effectiveness in elderly patients. In second line, the only therapy that showed efficacy also in this subgroup was afatinib, which, besides well tolerated and administered orally, did not show efficacy reduction in elderly population at the subgroup analysis of the study which compared it with methotrexate. However, $s$ already discussed above, the efficacy of afatinib was overall clinically modest, and the best treatment of these patients remains an umnet need.

There are still some doubts even about the use of immunotherapy in R/M HNSCC in clinical practice. It is undeniable that nivolumab gives a significant OS advantage compared with the existing alternatives in the second line setting, however it is also true that analyzing the PFS curves, in the first two months of therapy they are substantially overlapping. This means that immunotherapy is not effective in reducing the risk of progression and worsening in the first months of second-line therapy. If in other neoplasms an initial "pseudo-progression" may be acceptable, in view of a continuation of therapy and a significant overall survival benefit, in head-neck cancers often a progressive disease can mean a relapse in the face or in the neck, all areas that often compromise the quality of life of the patient so much to compromise the continuation of any therapy, and thus determining the definitive loss of the patient. If the results of pembrolizumab will be confirmed by the definitive paper, and they will consist of the typical pattern of immunotherapy response, this problem will become even more evident. It would be 
desirable in the future to find a second line of therapy capable not only of increasing overall survival, but also of reducing the risk of recurrence especially during the first months of treatment. Naturally, when these drugs will arrive even in first line, as anticipated by KEYNOTE-048 study, these problems will become even more relevant, as well as the considerations about health-economics.

In conclusion, R/M HNSCC surely represent an insidious and complex group of diseases, in which most of the strategies tested are destined to fail. Nevertheless, given the increasing incidence and severity of disease by the clinical point of view, it is mandatory to continue the efforts to find new therapeutic weapons that can help clinicians to face this difficult challenge.

\section{Conflict of interest statement}

We declare to have no conflict of interest.

\section{References}

1. Ferlay, J., et al. "GLOBOCAN, Cancer incidence and mortality worldwide: IARC CancerBase No. 10 [Internet]. Lyon, France: International Agency for Research on Cancer; 2010." globocan. iarc. fr (2012).

2. Grégoire, Vincent, et al. "Squamous cell carcinoma of the head and neck: EHNSESMO-ESTRO Clinical Practice Guidelines for diagnosis, treatment and follow-up." Annals of oncology 21.suppl 5 (2010): v184-v186.

3. Vermorken, Jan B., et al. "Open-Label, Uncontrolled, Multicenter Phase II Study to Evaluate the Efficacy and Toxicity of Cetuximab As a Single Agent in Patients With Recurrent and/or Metastatic Squamous Cell Carcinoma of the." Journal of clinical oncology 25.16 (2007): 2171-2177.

4. Baselga, José, et al. "Phase II multicenter study of the antiepidermal growth factor receptor monoclonal antibody cetuximab in combination with platinum-based chemotherapy in patients with platinum-refractory metastatic and/or recurrent squamous cell carcinoma of the head and neck." Journal of Clinical Oncology 23.24 (2005): 55685577.

5. Herbst, Roy S., et al. "Phase II multicenter study of the epidermal growth factor receptor antibody cetuximab and cisplatin for recurrent and refractory squamous cell carcinoma of the head and neck." Journal of Clinical Oncology 23.24 (2005): 5578-5587. 
6. Burtness, Barbara, et al. "Phase III randomized trial of cisplatin plus placebo compared with cisplatin plus cetuximab in metastatic/recurrent head and neck cancer: an Eastern Cooperative Oncology Group study." Journal of Clinical Oncology 23.34 (2005): 8646-8654.

7. Vermorken, Jan B., et al. "Platinum-based chemotherapy plus cetuximab in head and neck cancer." New England Journal of Medicine 359.11 (2008): 1116-1127.

8. Hitt, R., et al. "Phase II study of the combination of cetuximab and weekly paclitaxel in the first-line treatment of patients with recurrent and/or metastatic squamous cell carcinoma of head and neck." Annals of Oncology 23.4 (2011): 1016-1022.

9. Knoedler, M., et al. "Phase II study of cetuximab in combination with docetaxel in patients with recurrent and/or metastatic squamous cell carcinoma of the head and neck after platinum-containing therapy: a multicenter study of the Arbeitsgemeinschaft Internistische Onkologie." Oncology 84.5 (2013): 284-289.

10. Guigay, J., et al. "Cetuximab, docetaxel, and cisplatin as first-line treatment in patients with recurrent or metastatic head and neck squamous cell carcinoma: a multicenter, phase II GORTEC study." Annals of Oncology 26.9 (2015): 1941-1947.

11. Clark, Joseph I., et al. "Phase I pilot study of oxaliplatin, infusional 5-FU, and cetuximab in recurrent or metastatic head and neck cancer." Medical Oncology 30.1 (2013): 358.

12. Vermorken, J. B., et al. "Phase II study of pemetrexed in combination with cisplatin and cetuximab in recurrent or metastatic squamous cell carcinoma of the head and neck." European Journal of Cancer 49.13 (2013): 2877-2883.

13. Vermorken, Jan B., et al. "Cisplatin and fluorouracil with or without panitumumab in patients with recurrent or metastatic squamous-cell carcinoma of the head and neck (SPECTRUM): an open-label phase 3 randomised trial." The lancet oncology 14.8 (2013): 697-710.

14. Wirth, Lori J., et al. "PARTNER: An open-label, randomized, phase 2 study of docetaxel/cisplatin chemotherapy with or without panitumumab as first-line treatment for recurrent or metastatic squamous cell carcinoma of the head and neck." Oral oncology 61 (2016): 31-40.

15. Rischin, Danny, et al. "PRISM: Phase 2 trial with panitumumab monotherapy as second-line treatment in patients with recurrent or metastatic squamous cell carcinoma of the head and neck." Head \& neck 38.S1 (2016). 
16. Siano, Marco, et al. "Multicenter Phase II Study of Panitumumab in Platinum Pretreated, Advanced Head and Neck Squamous Cell Cancer." The Oncologist (2017): theoncologist-2017.

17. Cohen, Ezra EW, et al. "Phase II trial of ZD1839 in recurrent or metastatic squamous cell carcinoma of the head and neck." Journal of clinical oncology 21.10 (2003): 1980-1987.

18. Cohen, Ezra EW, et al. "Phase II trial of gefitinib $250 \mathrm{mg}$ daily in patients with recurrent and/or metastatic squamous cell carcinoma of the head and neck." Clinical Cancer Research 11.23 (2005): 8418-8424.

19. Kirby, A. M., et al. "Gefitinib (ZD1839, Iressa ${ }^{\mathrm{TM}}$ ) as palliative treatment in recurrent or metastatic head and neck cancer." British journal of cancer 94.5 (2006): 631-636.

20. Perez, Cesar A., et al. "Phase II study of gefitinib adaptive dose escalation to skin toxicity in recurrent or metastatic squamous cell carcinoma of the head and neck." Oral oncology 48.9 (2012): 887-892.

21. Kushwaha, Vandana Singh, et al. "Gefitinib, methotrexate and methotrexate plus 5fluorouracil as palliative treatment in recurrent head and neck squamous cell carcinoma." Cancer biology \& therapy 16.2 (2015): 346-351.

22. Stewart, J. Simon W., et al. "Phase III study of gefitinib compared with intravenous methotrexate for recurrent squamous cell carcinoma of the head and neck." Journal of clinical oncology 27.11 (2009): 1864-1871.

23. Argiris, Athanassios, et al. "Phase III randomized, placebo-controlled trial of docetaxel with or without gefitinib in recurrent or metastatic head and neck cancer: an eastern cooperative oncology group trial." Journal of Clinical Oncology 31.11 (2013): 14051414.

24. Soulieres, Denis, et al. "Multicenter phase II study of erlotinib, an oral epidermal growth factor receptor tyrosine kinase inhibitor, in patients with recurrent or metastatic squamous cell cancer of the head and neck." Journal of clinical oncology 22.1 (2004): 7785.

25. Siu, Lillian L., et al. "Phase I/II trial of erlotinib and cisplatin in patients with recurrent or metastatic squamous cell carcinoma of the head and neck: a Princess Margaret Hospital phase II consortium and National Cancer Institute of Canada Clinical Trials Group Study." Journal of Clinical Oncology 25.16 (2007): 2178-2183. 
26. Cohen, Ezra EW, et al. "Erlotinib and bevacizumab in patients with recurrent or metastatic squamous-cell carcinoma of the head and neck: a phase I/II study." The lancet oncology 10.3 (2009): 247-257.

27. De Souza, Jonas A., et al. "A phase II study of lapatinib in recurrent/metastatic squamous cell carcinoma of the head and neck." Clinical Cancer Research 18.8 (2012): 2336-2343.

28. Machiels, Jean-Pascal, et al. "Zalutumumab plus best supportive care versus best supportive care alone in patients with recurrent or metastatic squamous-cell carcinoma of the head and neck after failure of platinum-based chemotherapy: an open-label, randomised phase 3 trial." The lancet oncology 12.4 (2011): 333-343.

29. Saloura, Vassiliki, et al. "An open-label single-arm, phase II trial of zalutumumab, a human monoclonal anti-EGFR antibody, in patients with platinum-refractory squamous cell carcinoma of the head and neck." Cancer chemotherapy and pharmacology 73.6 (2014): 1227-1239.

30. Jimeno, Antonio, et al. "Phase lb study of duligotuzumab (MEHD7945A) plus cisplatin/5-fluorouracil or carboplatin/paclitaxel for first-line treatment of recurrent/metastatic squamous cell carcinoma of the head and neck." Cancer 122.24 (2016): 3803-3811.

31. Abdul Razak, A. R., et al. "A phase II trial of dacomitinib, an oral pan-human EGF receptor (HER) inhibitor, as first-line treatment in recurrent and/or metastatic squamouscell carcinoma of the head and neck." Annals of oncology 24.3 (2012): 761-769.

32. Kim, Han Sang, et al. "Phase II clinical and exploratory biomarker study of dacomitinib in patients with recurrent and/or metastatic squamous cell carcinoma of head and neck." Clinical Cancer Research (2014): clincanres-1756.

33. Seiwert, T. Y., et al. "A randomized, phase II study of afatinib versus cetuximab in metastatic or recurrent squamous cell carcinoma of the head and neck." Annals of oncology 25.9 (2014): 1813-1820.

34. Machiels, Jean-Pascal H., et al. "Afatinib versus methotrexate as second-line treatment in patients with recurrent or metastatic squamous-cell carcinoma of the head and neck progressing on or after platinum-based therapy (LUX-Head \& Neck 1): an openlabel, randomised phase 3 trial." The Lancet Oncology 16.5 (2015): 583-594.

35. Clement, Paul M., et al. "Afatinib versus methotrexate in older patients with secondline recurrent and/or metastatic head and neck squamous cell carcinoma: subgroup analysis of the LUX-Head \& Neck 1 trial." Annals of Oncology 27.8 (2016): 1585-1593. 
36. Elser, Christine, et al. "Phase II trial of sorafenib in patients with recurrent or metastatic squamous cell carcinoma of the head and neck or nasopharyngeal carcinoma." Journal of Clinical Oncology 25.24 (2007): 3766-3773.

37. Williamson, Stephen K., et al. "Phase II evaluation of sorafenib in advanced and metastatic squamous cell carcinoma of the head and neck: Southwest Oncology Group Study S0420." Journal of Clinical Oncology 28.20 (2010): 3330-3335.

38. Thomson, David J., et al. "Phase II trial of sorafenib in advanced salivary adenoid cystic carcinoma of the head and neck." Head \& neck 37.2 (2015): 182-187.

39. Lalami, Yassine, et al. "Phase II trial evaluating the efficacy of sorafenib (BAY 439006) and correlating early fluorodeoxyglucose positron emission tomography-CT response to outcome in patients with recurrent and/or metastatic head and neck cancer." Head \& neck 38.3 (2016): 347-354.

40. Gilbert, Jill, et al. "A randomized phase II efficacy and correlative studies of cetuximab with or without sorafenib in recurrent and/or metastatic head and neck squamous cell carcinoma." Oral oncology 51.4 (2015): 376-382.

41. Argiris, Athanassios, et al. "Phase II trial of pemetrexed and bevacizumab in patients with recurrent or metastatic head and neck cancer." Journal of Clinical Oncology 29.9 (2011): 1140-1145.

42. Argiris, A., et al. "Cetuximab and bevacizumab: preclinical data and phase II trial in recurrent or metastatic squamous cell carcinoma of the head and neck." Annals of oncology 24.1 (2012): 220-225.

43. Machiels, Jean-Pascal H., et al. "Phase II study of sunitinib in recurrent or metastatic squamous cell carcinoma of the head and neck: GORTEC 2006-01." Journal of Clinical Oncology 28.1 (2009): 21-28.

44. Fountzilas, George, et al. "A phase II study of sunitinib in patients with recurrent and/or metastatic non-nasopharyngeal head and neck cancer." Cancer chemotherapy and pharmacology 65.4 (2010): 649-660.

45. Choong, Nicholas W., et al. "Phase II study of sunitinib malate in head and neck squamous cell carcinoma." Investigational new drugs 28.5 (2010): 677-683.

46. Cooney, Matthew M., et al. "A phase IB clinical and pharmacokinetic study of the angiogenesis inhibitor SU5416 and paclitaxel in recurrent or metastatic carcinoma of the head and neck." Cancer chemotherapy and pharmacology 55.3 (2005): 295-300.

47. Fury, Matthew G., et al. "A Phase II study of SU5416 in patients with advanced or recurrent head and neck cancers." Investigational new drugs 25.2 (2007): 165-172. 
48. Swiecicki, Paul L., et al. "A phase II study evaluating axitinib in patients with unresectable, recurrent or metastatic head and neck cancer." Investigational new drugs 33.6 (2015): 1248-1256.

49. Limaye, Sewanti, et al. "A randomized phase II study of docetaxel with or without vandetanib in recurrent or metastatic squamous cell carcinoma of head and neck (SCCHN)." Oral oncology 49.8 (2013): 835-841.

50. Fury, Matthew G., et al. "A phase I study of daily everolimus plus low-dose weekly cisplatin for patients with advanced solid tumors." Cancer chemotherapy and pharmacology 69.3 (2012): 591-598.

51. Saba, Nabil F., et al. "Phase 1 and pharmacokinetic study of everolimus in combination with cetuximab and carboplatin for recurrent/metastatic squamous cell carcinoma of the head and neck." Cancer 120.24 (2014): 3940-3951.

52. Massarelli, E., et al. "Phase II trial of everolimus and erlotinib in patients with platinum-resistant recurrent and/or metastatic head and neck squamous cell carcinoma." Annals of Oncology 26.7 (2015): 1476-1480.

53. Fury, Matthew G., et al. "A phase I study of temsirolimus plus carboplatin plus paclitaxel for patients with recurrent or metastatic $(R / M)$ head and neck squamous cell cancer (HNSCC)." Cancer chemotherapy and pharmacology 70.1 (2012): 121-128.

54. Bauman, Julie E., et al. "A phase II study of temsirolimus and erlotinib in patients with recurrent and/or metastatic, platinum-refractory head and neck squamous cell carcinoma." Oral oncology 49.5 (2013): 461-467.

55. Grünwald, V., et al. "TEMHEAD: a single-arm multicentre phase II study of temsirolimus in platin-and cetuximab refractory recurrent and/or metastatic squamous cell carcinoma of the head and neck (SCCHN) of the German SCCHN Group (AIO)." Annals of Oncology 26.3 (2014): 561-567.

56. Bowles, Daniel W., et al. "A multicenter phase 1 study of PX-866 and cetuximab in patients with metastatic colorectal carcinoma or recurrent/metastatic squamous cell carcinoma of the head and neck." Investigational new drugs 32.6 (2014): 1197-1203.

57. Jimeno, A., et al. "A randomized, phase II trial of cetuximab with or without PX-866, an irreversible oral phosphatidylinositol 3-kinase inhibitor, in patients with relapsed or metastatic head and neck squamous cell cancer." Annals of Oncology 26.3 (2014): 556561. 
58. Jimeno, Antonio, et al. "A randomized, phase 2 trial of docetaxel with or without PX866, an irreversible oral phosphatidylinositol 3-kinase inhibitor, in patients with relapsed or metastatic head and neck squamous cell cancer." Oral oncology 51.4 (2015): 383-388.

59. Ando, Yuichi, et al. "Phase I dose-escalation study of buparlisib (BKM120), an oral pan-class I PI3K inhibitor, in Japanese patients with advanced solid tumors." Cancer science 105.3 (2014): 347-353.

60. Soulières, Denis, et al. "Buparlisib and paclitaxel in patients with platinumpretreated recurrent or metastatic squamous cell carcinoma of the head and neck (BERIL1): a randomised, double-blind, placebo-controlled phase 2 trial." The Lancet Oncology 18.3 (2017): 323-335.

61. Michel, Loren, et al. "Phase I trial of palbociclib, a selective cyclin dependent kinase 4/6 inhibitor, in combination with cetuximab in patients with recurrent/metastatic head and neck squamous cell carcinoma." Oral oncology 58 (2016): 41-48.

62. Chung, C. H., et al. "Nuclear factor-kappa B pathway and response in a phase II trial of bortezomib and docetaxel in patients with recurrent and/or metastatic head and neck squamous cell carcinoma." Annals of Oncology 21.4 (2009): 864-870.

63. Gilbert, Jill, et al. "Phase II 2-arm trial of the proteasome inhibitor, PS-341 (bortezomib) in combination with irinotecan or PS-341 alone followed by the addition of irinotecan at time of progression in patients with locally recurrent or metastatic squamous cell carcinoma of the head and neck (E1304): A trial of the Eastern Cooperative Oncology Group." Head \& neck 35.7 (2013): 942-948.

64. Smith, David C., et al. "Phase 1 study of ixazomib, an investigational proteasome inhibitor, in advanced non-hematologic malignancies." Investigational new drugs 33.3 (2015): 652-663.

65. Brooks, Heather D., et al. "Phase 2 study of dasatinib in the treatment of head and neck squamous cell carcinoma." Cancer 117.10 (2011): 2112-2119.

66. Seiwert, Tanguy, et al. "Phase II trial of single-agent foretinib (GSK1363089) in patients with recurrent or metastatic squamous cell carcinoma of the head and neck." Investigational new drugs 31.2 (2013): 417-424.

67. Haigentz, Missak, et al. "Phase II trial of the histone deacetylase inhibitor romidepsin in patients with recurrent/metastatic head and neck cancer." Oral oncology 48.12 (2012): 1281-1288. 
68. Blumenschein, George R., et al. "Phase II trial of the histone deacetylase inhibitor vorinostat (Zolinza ${ }^{\mathrm{TM}}$, suberoylanilide hydroxamic acid, SAHA) in patients with recurrent and/or metastatic head and neck cancer." Investigational new drugs 26.1 (2008): 81-87.

69. Ruzsa, A., et al. "Phase 2, open-label, 1: 1 randomized controlled trial exploring the efficacy of EMD 1201081 in combination with cetuximab in second-line cetuximab-naive patients with recurrent or metastatic squamous cell carcinoma of the head and neck (R/M SCCHN)." Investigational new drugs 32.6 (2014): 1278-1284.

70. Machiels, Jean-Pascal, et al. "Phase Ib trial of the Toll-like receptor 9 agonist IMO2055 in combination with 5-fluorouracil, cisplatin, and cetuximab as first-line palliative treatment in patients with recurrent/metastatic squamous cell carcinoma of the head and neck." Investigational new drugs 31.5 (2013): 1207-1216.

71. Riechelmann, Herbert, et al. "Phase I trial with the CD44v6-targeting immunoconjugate bivatuzumab mertansine in head and neck squamous cell carcinoma." Oral oncology 44.9 (2008): 823-829.

72. Carducci, Michael A., et al. "Phase I dose escalation and pharmacokinetic study of enzastaurin, an oral protein kinase C beta inhibitor, in patients with advanced cancer." Journal of Clinical Oncology 24.25 (2006): 4092-4099.

73. Tang, Patricia A., et al. "Phase II study of ispinesib in recurrent or metastatic squamous cell carcinoma of the head and neck." Investigational new drugs 26.3 (2008): 257-264.

74. Hanrahan, Emer O., et al. "A phase II study of Lonafarnib (SCH66336) in patients with chemorefractory, advanced squamous cell carcinoma of the head and neck." American journal of clinical oncology 32.3 (2009): 274-279.

75. Fury, Matthew G., et al. "Phase II study of saracatinib (AZD0530) for patients with recurrent or metastatic head and neck squamous cell carcinoma (HNSCC)." Anticancer research 31.1 (2011): 249-253.

76. Schmitz, Sandra, et al. "Phase II study of figitumumab in patients with recurrent and/or metastatic squamous cell carcinoma of the head and neck: clinical activity and molecular response (GORTEC 2008-02)." Annals of oncology 23.8 (2012): 2153-2161.

77. Keam, Bhumsuk, et al. "Phase 2 study of dovitinib in patients with metastatic or unresectable adenoid cystic carcinoma." Cancer 121.15 (2015): 2612-2617.

78. Jimeno $A$, et al. "A phase 2 study of dalantercept, an activin receptor-like kinase-1 ligand trap, in patients with recurrent or metastatic squamous cell carcinoma of the head and neck." Cancer 122.23 (2016): 3641-3649. 
79. Kirkwood, John M., et al. "Immunotherapy of cancer in 2012." CA: a cancer journal for clinicians 62.5 (2012): 309-335.

80. Brahmer, Julie R., et al. "Phase I study of single-agent anti-programmed death-1 (MDX-1106) in refractory solid tumors: safety, clinical activity, pharmacodynamics, and immunologic correlates." Journal of clinical oncology 28.19 (2010): 3167.

81. Gettinger, Scott N., et al. "Overall survival and long-term safety of nivolumab (antiprogrammed death 1 antibody, BMS-936558, ONO-4538) in patients with previously treated advanced non-small-cell lung cancer." Journal of clinical oncology 33.18 (2015): 2004.

82. Topalian, Suzanne L., et al. "Safety, activity, and immune correlates of anti-PD-1 antibody in cancer." New England Journal of Medicine 366.26 (2012): 2443-2454.

83. Ferris, Robert L., et al. "Nivolumab for recurrent squamous-cell carcinoma of the head and neck." N Engl J Med 2016.375 (2016): 1856-1867.

84. Seiwert, Tanguy Y., et al. "Safety and clinical activity of pembrolizumab for treatment of recurrent or metastatic squamous cell carcinoma of the head and neck (KEYNOTE-012): an open-label, multicentre, phase 1b trial." The lancet oncology 17.7 (2016): 956-965.

85. Bauml, Joshua, et al. "Pembrolizumab for platinum-and cetuximab-refractory head and neck cancer: results from a single-arm, phase II study." Journal of Clinical Oncology 35.14 (2017): 1542-1549.

86. Cohen, E. E., et al. "LBA45_PRPembrolizumab (pembro) vs standard of care (SOC) for recurrent or metastatic head and neck squamous cell carcinoma (R/M HNSCC): Phase 3 KEYNOTE-040 trial." Annals of Oncology 28. suppl_5 (2017). KEYNOTE-040 trial." Ann Oncol. (2017) 28:mdx440.040 10.1093/annonc/mdx440.040.

87. Segal SIO NH, et al. "Clinical activity and safety of MEDI4736, an anti-PD-L1 antibody, In Head and neck cancer." ESMO 2016 Congress.

88. Zandberg $D$ et al. "Durvalumab for recurrent/metastatic (R/M) head and neck squamous cell carcinoma (HNSCC): preliminary results from a single-arm, phase 2 study." ESMO 2017 Congress.

89. Simo, R., Robinson, M., Lei, M., Sibtain, A., \& Hickey, S. (2016). Nasopharyngeal carcinoma: United Kngdom national multidisciplinary guidelines. The Journal of Laryngology \& Otology, 130(S2), S97-S103. 
90. Chan, Anthony TC, et al. "Multicenter, phase II study of cetuximab in combination with carboplatin in patients with recurrent or metastatic nasopharyngeal carcinoma." Journal of clinical oncology 23.15 (2005): 3568-3576.

91. Chua, Daniel TT, et al. "Phase II study of gefitinib for the treatment of recurrent and metastatic nasopharyngeal carcinoma." Head \& neck 30.7 (2008): 863-867.

92. Sood, S., M. McGurk, and F. Vaz. "Management of salivary gland tumours: United Kingdom national multidisciplinary guidelines." The Journal of Laryngology \& Otology 130.S2 (2016): S142-S149.

93. Locati, L. D., et al. "Cetuximab in recurrent and/or metastatic salivary gland carcinomas: a phase II study." Oral oncology 45.7 (2009): 574-578.

94. Jakob, John A., et al. "Phase II study of gefitinib in patients with advanced salivary gland cancers." Head \& neck 37.5 (2015): 644-649.

95. Locati, L. D., et al. "A phase II study of sorafenib in recurrent and/or metastatic salivary gland carcinomas: Translational analyses and clinical impact." European Journal of Cancer 69 (2016): 158-165.

96. Kim, Youjin, et al. "Clinical trial of nintedanib in patients with recurrent or metastatic salivary gland cancer of the head and neck: A multicenter phase 2 study (Korean Cancer Study Group HN14-01)." Cancer 123.11 (2017): 1958-1964.

97. Argiris, Athanassios, et al. "A phase 2 trial of bortezomib followed by the addition of doxorubicin at progression in patients with recurrent or metastatic adenoid cystic carcinoma of the head and neck." Cancer 117.15 (2011): 3374-3382.

98. Wong, S. J., et al. "Phase II trial of dasatinib for recurrent or metastatic C-KIT expressing adenoid cystic carcinoma and for nonadenoid cystic malignant salivary tumors." Annals of Oncology 27.2 (2015): 318-323.

99. Pfeffer, M. Raphael, et al. "A phase II study of Imatinib for advanced adenoid cystic carcinoma of head and neck salivary glands." Oral oncology 43.1 (2007): 33-36.

100. Drilon, Alexander, et al. "Efficacy of larotrectinib in TRK fusion-positive cancers in adults and children." New England Journal of Medicine 378.8 (2018): 731-739. 